\title{
A SIMPLE AND FAST NUMERICAL METHOD FOR THE COMPUTATION OF DAILY TOTALS OF CROP PHOTOSYNTHESIS
}

\section{J. GOUDRIAAN}

Department of Theoretical Production Ecology, Agricultural University, PO Box 430 6700 AK, Wageningen (The Netherlands)

(Received October 24, 1985; revision accepted May 13, 1986)

\section{ABSTRACT}

Goudriaan, J., 1986. A simple and fast numerical method for the computation of daily totals of crop photosynthesis. Agric. For Meteorol., 38: 257-262.

Gaussian integration provides an accurate, fast and flexible method to calculate instantaneous or daily crop photosynthesis. The three-point method combines accuracy and speed, and it is so flexible that a depth or time dependent photosynthetic response curve can be easily introduced. Because of this efficiency it is an excellent method to be used in a subroutine in larger crop growth models.

\section{INTRODUCTION}

The rate of crop photosynthesis can be computed from the photosynthesislight response curve of individual leaves, the incoming radiation and the leaf area index. Leaf angle distribution, extinction and reflection coefficients must also be known since they influence the distribution of the available radiation. This computational problem was essentially solved by De Wit (1965). He applied a stratification of the leaf canopy, calculated the absorbed radiation and the corresponding rates of photosynthesis of sunlit and shaded leaves in each layer and added their contributions to find the rate of crop photosynthesis. This procedure was repeated every $15 \mathrm{~min}$ to obtain the daily total of crop photosynthesis. This procedure is lucid and flexible but rather time consuming. Therefore its use during an entire season, as one would want in simulation of crop growth, might become problematic.

For application in such models Goudriaan and Van Laar (1978) developed a summary model for the daily total of crop photosynthesis, based on a semi-empirical equation, fitted to computer output of the detailed model. This fast procedure was used in the simulation model SUCROS which operates with daily time intervals (Penning de Vries and Van Laar, 1982). However, the meaning of the coefficients in this semi-empirical equation is not clear in terms of the processes involved, so that it is practically impossible to adapt this equation for deviating circumstances. For instance, it 
$A=A_{m}$ eH/ $\left(A_{m}+C H\right)$

where $A_{\mathrm{m}}=$ maximum assimilation (saturated by radiation) with a value of $1000 \mu \mathrm{g} \mathrm{m}^{-2} \mathrm{~s}^{-1}$ and $\epsilon=$ initial light use efficiency with a value of $10 \mu \mathrm{g} \mathrm{J}^{-1}$. These values, which are typical for healthy leaves of $\mathrm{C}_{3}$ crop species, correspond to a $50 \%$ point of maximum assimilation at $100 \mathrm{~W} \mathrm{~m}^{-2}$ of absorbed photosynthetically active radiation (PAR). The hyperbolic shape is not necessarily the best representation of the light response curve, but at least it can be analytically integrated over the depth of the crop canopy. It is then possible to assess the accuracy of the Gaussian method without resorting to numerical theory. Another assumption, necessary for analytical integration, is that photosynthetically active radiation $H$ absorbed per leaf area decreases exponentially with leaf area depth $L$ as $k I_{0} \mathrm{e}^{-k L}$, where $I_{0}$ is the incoming flux of PAR at the top of the canopy, and $k$ its extinction coefficient. From top to bottom, $L$ ranges between zero and LAI.

TABLE I

Three-point Gaussian integration, and the analytical solution

Analytical solution of canopy assimilation, using eq. 1:

$\int_{0}^{\mathrm{LAI}} \frac{A_{\mathrm{m}} \epsilon k I_{0} \mathrm{e}^{-k L} d L}{A_{\mathrm{m}}+\epsilon k I_{0} \mathrm{e}^{-k L}}=\frac{A_{\mathrm{m}}}{k} \ln \left\{\frac{A_{\mathrm{m}}+\epsilon k I_{0}}{A_{\mathrm{m}}+\epsilon k I_{0} \mathrm{e}^{-k \cdot \mathrm{LAI}}}\right\}=843.74 \mu \mathrm{g} \mathrm{m}^{-2} \mathrm{~s}^{-1}$ Gaussian integration to find canopy assimilation:

$L$
$\quad A($ eq. 1$)$

$-1 \quad(0.5-\sqrt{0.15})$ LAI $=0.5635083 \quad 389.16898$

$0 \quad 0.5 \mathrm{LAI} \quad=2.5 \quad 119.2027$

$1 \quad(0.5+\sqrt{0.15}) \mathrm{LAI}=4.4364916 \quad 27.944458$

$$
\begin{aligned}
& 389.16898 \\
& \times 1.6=190.72432 \\
& \underline{27.944458}+ \\
& \frac{\mathrm{LAI}}{3.6} \times 607.837758=844.22
\end{aligned}
$$

Parameter values: $k=0.8, \mathrm{LAI}=5, I_{0}=125 \mathrm{~W} \mathrm{~m}^{-2}, A_{\mathrm{m}}=1000 \mu \mathrm{g} \mathrm{m}^{-2} \mathrm{~s}^{-1}, \epsilon=10$ $\mu \mathrm{g} \mathrm{J}^{-1}$

As shown in Table I, the result of the three-point Gaussian integration method deviates $<0.1 \%$ from the analytical solution. Therefore a higher order method, which is inevitably more complicated, is not necessary.

A depth dependent $A_{\mathrm{m}}$ can also be easily introduced. For example, when $A_{\mathrm{m}}$ decreases linearly with leaf area depth $L$ as:

$A_{\mathrm{m}}=1000(1-L / \mathrm{LAI})$

Three-point Gaussian integration gives $783.54 \mu \mathrm{g} \mathrm{m}^{-2} \mathrm{~s}^{-1}$. In this example an analytical solution is not available, so that a higher order numerical method must be used to find the answer with at least one order more accuracy. Such a method gives $782.03 \mu \mathrm{g} \mathrm{m}^{-2} \mathrm{~s}^{-1}$, showing that the three-point method is also accurate enough in this situation. 
value of the rate is taken halfway through the integration interval. As can be seen in Fig. 1, it gives a more accurate result than the rectangular integration method, because the errors left and right of the evaluation point in the centre practically cancel.

In a more formal analysis, which follows now, the integration interval is normalized to unity, and centralized between $x=-1 / 2$ and $x=1 / 2$. The polynomial given by $y=a+b x+c x^{2}+d x^{3} \ldots$ will have the value $a$ at the centre point $x=0$. Therefore the integrated value obtained by the one-point Gaussian integration, will also be equal to $a$. Analytical integration of the polynomial term by term shows that the integrals of all odd terms disappear because of the symmetry around $x=0$. That means that the one-point method not only exactly integrates $y=a$, but also $y=a+b x$. Similarly the two-point method (Fig. 1) will exactly integrate $y=a+b x+c x^{2}+d x^{3}$. The next step is the three-point method which will enable exact integration of the fourth order term (and automatically of the fifth as well). Due to the requirement of symmetry, one of the three function evaluation points will be at the centre, $x=0$. The other two will be located at either side, at a distance $\gamma$ from the centre. The function value at $x=0$ will be given a weight $w$, different from unity. The values of the relative distance $\gamma$ and of the weight $w$ can be derived from the requirement that both the second and the fourth order terms of the polynomial will have to be exactly integrated:

$$
\begin{gathered}
2^{\text {nd }} \text { order } \frac{(-\gamma)^{2}+0 * w+\gamma^{2}}{1+w+1}=\int_{-1 / 2}^{1 / 2} x^{2} d x=\frac{1}{12} \\
4^{\text {th }} \text { order } \frac{(-\gamma)^{4}+0 * w+\gamma^{4}}{1+w+1}=\int_{-1 / 2}^{1 / 2} x^{4} d x=\frac{1}{80}
\end{gathered}
$$

By taking the ratio of these two equations the relative distance $\gamma$ is found as $(0.15)^{1 / 2}$ and the weight $w$ was 1.6 .

\section{CANOPY PHOTOSYNTHESIS}

When applied to canopy photosynthesis, the integration interval must be taken as the leaf area index LAI. The rate of photosynthesis must be computed at three depths: in the middle, and at two levels at a relative distance of $(0.15)^{1 / 2}$ LAI from either side of the middle. The weight of the photosynthesis rate half way must be taken as 1.6 relative to those at either side.

In the following example the term assimilation $\left(\right.$ of $\left.\mathrm{CO}_{2}\right)$ is used instead of photosynthesis, because it is assimilation that is measured, not photo'synthesis. The physiological relation between these two processes is not considered here. The shape of the response curve of leaf assimilation $A$ to absorbed photosynthetically active radiation $H$ is assumed to be hyperbolic: 
For integration with respect to time a similar procedure can be followed. The radiation level will depend on solar height, and for modeling purposes it is supposed that it follows a smooth time course. As shown by Spitters et al. (1986) this assumption gives only a slight overestimation of crop photosynthesis as compared to the daily total when there are more realistic random fluctuations in irradiance. To permit analytical integration a simplified time dependence of irradiance is used:

$I_{0}=I_{\mathrm{m}} \sin \left(2 \pi\left(t_{\mathrm{h}}-6\right) / 24\right) \quad 6<t_{\mathrm{h}}<12$

where $I_{\mathrm{m}}=500 \mathrm{~W} \mathrm{~m}^{-2}$ and $t_{\mathrm{h}}$ indicates solar time in $\mathrm{h}$. The integral of irradiation over this period can be found analytically as $6 \times 2 / \pi \times 3600 \times$ $500=6.875494 \mathrm{MJ} \mathrm{m}^{-2}$. Gaussian integration gives $6.880127 \mathrm{MJ} \mathrm{m}^{-2}$, so that the deviation is only $0.07 \%$. When this sinusoidal time course of radiation is applied to a single leaf with a hyperbolic response function, the integral of photosynthesis can still be found analytically (Monteith, 1965). For the first half of the daylight period this yields $15.165 \mathrm{~g} \mathrm{~m}^{-2}$ (same leaf characteristics are used as before), whereas the three-point Gaussian integration gives $15.279 \mathrm{~g} \mathrm{~m}^{-2}$. The deviation is $0.7 \%$, which is ten times higher than for the plain sine wave, but it is still quite acceptable. Moreover, for this example the analytical solution is getting so complicated that it is not simpler to use than the Gaussian method. These examples indicate that the Gaussian method can also be used for integration over the diurnal course.

\section{APPLICATION IN LARGER MODELS}

In a model with time steps of one day these integrations can be done in a subroutine, with nested loops of three steps each. For instance one loop will step through the depth profile, and may be embedded within another loop that will step through time. In these simple models no distinction is made between morning and afternoon response, so that it suffices to compute the total of the first half of the day and double its value. For quite a number of applications the number of computations required is so small that the Gaussian method is feasible on a pocket calculator. Also this method is now implemented in SUCROS, and has been used to make sensitivity tests, such as presented by Spitters (1986).

\section{REFERENCES}

De Wit, C.T., 1965. Photosynthesis of leaf canopies. Agric. Res. Reports 663. Pudoc, Wageningen.

Goudriaan, J. and van Laar, H.H., 1978. Calculation of daily totals of the gross assimilation of leaf canopies. Neth. J. Agric. Sci., 26: 373-382.

Lanczos, C., 1957. Applied Analysis. Pitman, London.

Monteigth, J.L., 1965. Light distribution and photosynthesis in field crops. Ann. Bot., 29: $17-37$. 


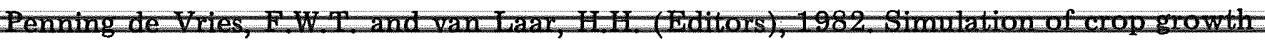
and plant production. Simulation Monograph, Pudoc, Wageningen.

Scheid, F., 1968. Numerical Analysis. Schaum's Outline Series. McGraw-Hill, London.

Spitters, C.J.T., 1986. Separating the diffuse and direct component of global radiation and its implications for modeling canopy photosynthesis. Part II. Calculation of canopy photosynthesis. Agric. For. Meteorol., 38: 239--250.

Spitters, C.J.T., Toussaint H.A.J.M. and Goudriaan, J., 1986. Separating the diffuse and direct component of global radiation and its implications for modeling canopy photosynthesis. Part I. Components of incoming radiation. Agric. For. Meteorol., 38: 225237. 